\title{
Addressing the physician-scientist pipeline: strategies to integrate research into clinical training programs
}

\author{
Sallie R. Permar, ${ }^{1,2,3}$ Rebecca A. Ward, ${ }^{4}$ Katherine J. Barrett, ${ }^{1,3}$ Stephanie A. Freel, ${ }^{1,3,5}$ Rasheed A. Gbadegesin, ${ }^{1,3,6}$ \\ Christopher D. Kontos, ${ }^{7}$ Patrick J. Hu, ${ }^{8}$ Katherine E. Hartmann, ${ }^{9}$ Christopher S. Williams, ${ }^{10}$ and Jatin M. Vyas ${ }^{4,11}$
}

'Department of Pediatrics, ${ }^{2}$ Duke Human Vaccine Institute, and ${ }^{3}$ Duke Office of Physician-Scientist Development, Duke University School of Medicine, Durham, North Carolina, USA. ${ }^{4}$ Division of Infectious Disease, Department of Medicine, Massachusetts Ceneral Hospital, Boston, Massachusetts, USA. ${ }^{5}$ Duke Office of Clinical Research, ${ }^{6}$ Duke Molecular Physiology Institute, and 'Division of Cardiology, Department of Medicine, Duke University Medical Center, Durham, North Carolina, USA. ${ }^{8}$ Division of Hematology and Oncology, ${ }^{9}$ Department of Obstetrics and Gynecology, and ${ }^{10}$ Division of Castroenterology, Hepatology, and Nutrition, Vanderbilt University School of Medicine, Nashville, Tennessee, USA. "Department of Medicine, Harvard Medical School, Boston, Massachusetts, USA.

\section{Introduction}

Physician-scientists are critical members of the biomedical workforce. The combination of rigorous scientific training and clinical skills uniquely positions them to bridge clinical needs with investigational pursuits by identifying important clinical questions that drive basic discoveries and translating those into therapeutics that improve patient outcomes. The impact of physician-scientists on biomedical science has been profound. Indeed, the recipients of the 2019 Nobel Prize in Physiology or Medicine, William Kaelin Jr., Gregg Semenza, and Peter Ratcliffe, are all physician-scientists. Furthermore, physician-scientists have been well represented as Nobel laureates (including Frederick Banting, Carl Cori, Earl Sutherland, Alfred Gilman, Michael Brown, Joseph Goldstein, Bernardo Houssay, Edwin Krebs, Peter Agre, Harold Varmus, Robert Lefkowitz, Brian Kobilka, and Ralph Steinman, to name a few). Yet, as the need for novel therapies has grown, the number of physician-scientists has declined (1). The reasons for this decline are numerous, including length and cost of clinical training, declining funding opportunities, reduced visibility of physician-scientist role models, and compensation disparities between academic and private practice careers. Residency, fellowship training, and entry into faculty - important entry points for budding physician-scientists and a period of continuity for those who wish to build on their earlier research experiences - are critical junctures where attrition occurs along the physician-scientist training timeline (2-5). Here, we describe innovative strategies implemented at three academic medical centers in the United States that are working to fill the physicianscientist pipeline. These strategies promote research during clinical training with an emphasis on cultivating intellectual curiosity, protecting research time, promoting physician-scientist leadership, tailoring mentor support, and fostering early career development, as well as providing financial resources to support trainees and young faculty. Additionally, we propose potential outcomes to measure the success of these strategies in strengthening the physician-scientist workforce.

\section{Structured paradigms}

for integrating physicianscientist development into clinical training

The loss of role models for clinical trainees negatively impacts their choice to participate in research $(1,5)$. Unless physician-scientists are part of academic medical center and medical education leadership, the development of future physician-scientists is unlikely to be a training priority. There are two emerging paradigms that promote institution-wide physician-scientist leadership: a centralized model and an integrated model, although these models are not mutually exclusive (Figure 1A).

The centralized model, as demonstrated by Duke University's Office of Physician-Scientist Development and Vanderbilt University's Office of Clinical and Translational Scientist Development, provides an institution-wide resource for phy-

Conflict of interest: The authors have declared that no conflict of interest exists.

Copyright: (c) 2020, American Society for Clinical Investigation.

Reference information: / Clin Invest. 2020;130(3):1058-1061. https://doi.org/10.1172/JCI136181.

sician-scientists and department-based programs to provide coordinated programming in career development and training efforts. The centralized model facilitates interactions among multiple departments, centers, and institutes to leverage institutional resources and invest in tailored programming. The Vanderbilt Physician Scientist Societies and affiliate programming (Medical Scientist Training Program, Harrison Society [Department of Medicine (DOM) ABIM Research Pathway], Newman Society [junior faculty development program]) and the Massachusetts General Hospital (MGH) DOM Stanbury Physician-Scientist Pathway are examples of an integrated model, which supports the development of physician-scientists throughout career transitions from graduate medical education to early and established faculty within each department. This model allows for sustained mentorship and training of physician-scientists. Although a perceived disadvantage of these institution-wide models might be an inability to provide individualized training, in fact, our collective experience has been that institution-wide programming allows leveraging of institutional resources to tailor mentoring to each trainee.

Physician-scientist leadership is essential and can be provided not only through institution-wide initiatives but also within specialties and during critical training periods, as demonstrated by MGH's DOM resident-focused approach. Residencyfocused models aim to provide directed support and mentorship in academic medicine to residents through a variety of mechanisms, as discussed below. A common thread throughout these three paradigms is integration of physician-scientists into leadership positions that set priorities 
A

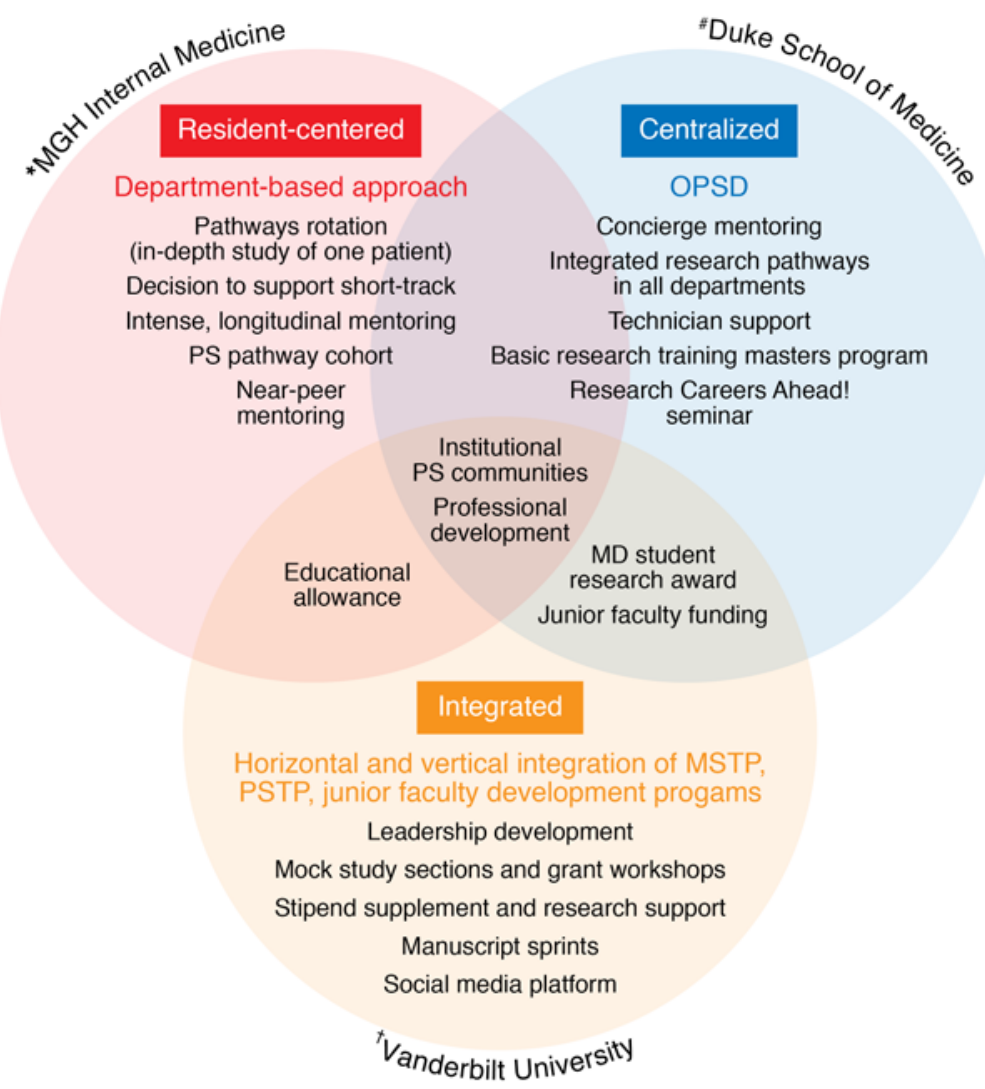

B

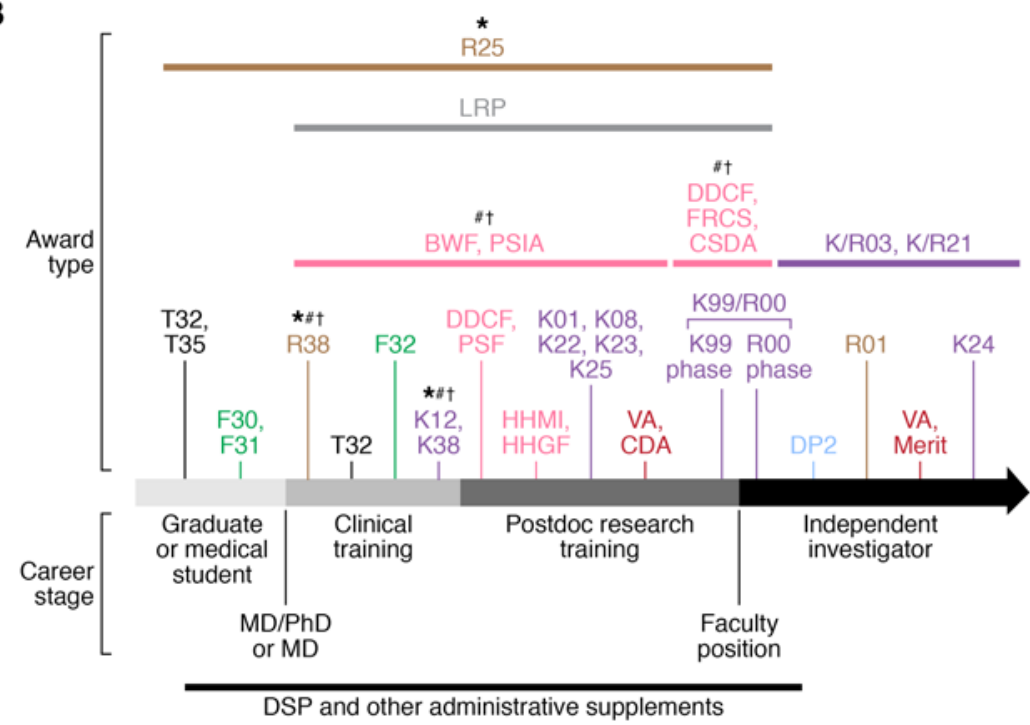

for medical education and research training, while also reflecting the diversity of trainees. Indeed, our institutions each have examples of physician-scientists serving as directors, associate deans, faculty directors, program managers, and residency program directors. Incorporating successful physician-scientists into clinical and research training leadership provides new trainees with strong role models, con- sistent guidance regarding research opportunities, and support for funded programs dedicated to physician-scientists.

\section{Key programming for physician-scientist trainees}

Specialty board-sanctioned research pathways provide a framework for integrating clinical and research training at academic medical centers. The persistent
Figure 1. Institutional approaches and funding opportunities for physician-scientists in training and the early career stage. (A) Structure and approaches taken by MCH Internal Medicine, Duke University School of Medicine (centralized in the Office of Physician Scientist Development [OPSD]), and Vanderbilt University School of Medicine to train and support new physician-scientists. (B) Funding from the NIH, the US Department of Veterans Affairs (VA), the Burroughs Wellcome Fund (BWF), the Doris Duke Charitable Foundation (DDCF), and Howard Hughes Medical Institute (HHMI). These mechanisms include the PhysicianScientist Institutional Award (PSIA), the Physician Scientist Fellowship (PSF), the Loan Repayment Program (LRP), the Fund to Retain Clinical Scientists (FRCS), and the Career Development Award (CDA). Symbols highlight mechanisms used to fund the institutional physicianscientist programs featured in this perspective. CSDA, Clinical Scientist Development Award: DSP, Diversity Supplement; HHGF, Hannah H. Gray fellows; MSTP, Medical Scientist Training Program; PS, physician-scientist. Adapted with permission from Dr. Shawn Gaillard (NIH). paucity of physician-scientists engaged in research-intensive careers at the conclusion of training underscores the need for institutions to develop additional programs to enhance professional research development. Mentorship during both residency and fellowship is critical to prepare trainees for success as they transition to faculty. Dedicated physician-scientist training programs (PSTPs) offer several 
advantages to trainees over standard residency and fellowship programs, including direct mentorship by active physicianscientists overseeing these programs. If developed appropriately, physician-scientist communities created by PSTPs catalyze peer-peer and near-peer mentoring relationships that facilitate the dissemination of critical stage-specific information through seminar series, social events, and career development workshops. Moreover, many PSTPs function as de facto "farm teams" for institutional faculty recruitment. Inclusion of alumni who have successfully transitioned to junior faculty in the institutional PSTP community (e.g., Vanderbilt's Harrison Society, Duke's Robert J. Lefkowitz Society, and MGH's Jackson Society) enriches training experiences through vertical integration across career stages, increasing opportunities for intrainstitutional networking.

Specific program features can significantly contribute to physician-scientist training success. During residency, structured curricula help maintain scientific curiosity despite time-intensive clinical obligations (e.g., MGH DOM Pathways Initiative) $(1,3)$. Protected research time during a fellowship is critical to provide an immersive research experience for trainees so they can begin establishing independent lines of scientific investigation. Benefits such as salary supplementation and child care disincentivize moonlighting and reduce the pressure to pursue careers in private practice. Activities including career trajectory and scholarly goal planning and focused grant-writing programs and grant concept reviews, coupled with financial resources (e.g., research stipends, technical support grants, pilot project grants) facilitate the acquisition of research funding. Mentoring committees composed of established physician-scientists ensure that trainees receive feedback on their research progress at regular intervals throughout their training and pair seamlessly with supplemental programs (e.g., career development consultation, curriculum vitae/ biosketch clinics, community-building activities). Dissemination of such programs throughout academic medical centers will likely contribute to increased retention of trainees in the physicianscientist pipeline.

\section{Funding opportunities for trainees and transition to early career faculty}

Sustaining successful physician-scientist pipelines requires a funding strategy that includes coverage for (a) protected time for both research and professional training; (b) research funding for scholars at various points in their career development; and (c) administrative support for mentorship, training, and evaluation. MGH, Duke, and Vanderbilt have used a variety of funding mechanisms to achieve these goals. NIH and VA funding opportunities provide research and training support for premedical, medical, and graduate students (T32, R25, F30/31), residents and fellows (T32, F32, R25, R38, K38, VA-CDA), and fellows/ early career faculty (K12/KL2) (Figure 1B). These NIH-funded mechanisms aim to support research education activities (R25), stimulate access to research during residency (R38), and enhance mentored research training opportunities (T32, K12, K38). These mechanisms are not available through all $\mathrm{NIH}$ institutes; specifically, the R38 is currently offered by only four institutes. Structural and administrative support to build professional development programs and administer internal research funding programs has largely been funded internally through medical school deans' offices, departments, and endowments and through large institutional grants (e.g., the Burroughs Wellcome Fund Physician-Scientist Institutional Award). Foundations such as the Doris Duke Charitable Foundation may be engaged to retain physician-scientists at early career faculty stages, when many struggle with balancing clinical, research, and home life responsibilities. Additional opportunities are offered by the Howard Hughes Medical Institute to promote diversity among physician-scientist trainees. Finally, for trainees who devote at least $50 \%$ of their efforts to research, the NIH Loan Repayment Program can offer a reduction in educational debt, and applications to this program should be highly encouraged beginning in residency. Overall, we have found that a strong and diverse portfolio of funding is necessary and that a variety of external opportunities exist to tackle the problem of a shrinking physician-scientist pipeline. Ultimately, institutional dedication to supporting trainees in pursuing these awards is critical to the success of a sustainably funded physician-scientist training program.

\section{Metrics of success}

Collecting metrics that demonstrate outcomes of institutional physician-scientist development programs will be important to inform the best use of limited resources to support these programs over the long term. Standard measures of success of scientific training programs typically include numbers and, more important, the impact of manuscripts, abstract presentations, grants applied for and received, awards received, and the proportion of graduates who stay in academic positions. However, additional metrics that may be harder to track are nonetheless vital to determining an institutional program's success, including the number of successful K-to-R grant transitions and the proportion of the program graduates' effort committed to research in early and later faculty years. Moreover, nonresearch factors (i.e., impact of educational debt, work-life integration, and physician burnout) should be followed and compared with those in primarily clinical careers. The development and maintenance of robust physician-scientist pathways can be framed as a strategy to diversify and strengthen the physician workforce and improve the health of the population, which should be a priority for both health systems and medical schools.

\section{Conclusions}

The decline in the physician-scientist pipeline is increasingly evident. We have described potential solutions to address this decline by incorporating physicianscientists into clinical and research training program leadership, establishing key programming during training, providing funding mechanisms to assist with research support, and creating new opportunities for professional development. Our programs represent three distinct but complementary approaches to provide robust physicianscientist development during clinical training, when it is often ignored. Broader implementation of such potential solutions will strengthen the physician-scientist workforce and revitalize this important component of academic medicine.

\section{Acknowledgments}

The authors would like to thank Katrina Armstrong (Physician-in-Chief, Depart- 
ment of Medicine, MGH); Nancy Brown (Chair, Department of Medicine, Vanderbilt University School of Medicine); Mary Klotman (Dean, Duke University School of Medicine); and Jeffrey Balser (Dean, Vanderbilt University School of Medicine) for their thoughtful advice and support. This work was supported in part by NIH grants (R25AI147393, to JMV; R38AI140297 and R38HL143612, to SRP; and R38HL143619, to CSW) and a Burroughs Wellcome Fund Physician-Scientist Institutional Award (PSIA) (1018899, to RAG).
Address correspondence to: Jatin M. Vyas, Department of Medicine, Massachusetts General Hospital, 55 Fruit Street, GrayBigelow Building 7, Room 746, Boston, Massachusetts 02114, USA.Email:jvyas@mgh. harvard.edu. Or to: Sallie R Permar, Duke Human Vaccine Institute, School of Medicine, Duke University, 2 Genome Court, MSRBII, 103020, Durham, North Carolina 27710, USA. Email: sallie.permar@ duke.edu.

1. Jain MK, Cheung VG, Utz PJ, Kobilka BK, Yamada $\mathrm{T}$, Lefkowitz R. Saving the endangered physician- scientist - a plan for accelerating medical breakthroughs. N Engl JMed. 2019;381(5):399-402.

2. Singh U, et al. Policy recommendations for optimizing the infectious diseases physicianscientist workforce. JInfect Dis. 2018;218(suppl_1):S49-S54.

3. Vyas JM, et al. The great opportunity: cultivating scientific inquiry in medical residency. J Infect Dis. 2018;218(suppl_1):S44-S48.

4. Hurst JH, et al. Cultivating research skills during clinical training to promote pediatric-scientist development. Pediatrics. 2019;144(2):e20190745.

5. Williams CS, et al. Training the physicianscientist: views from program directors and aspiring young investigators. JCI Insight. 2018;3(23):125651. 